\title{
IDEMPOTENT MEASURES ON COMPACT SEMIGROUPS
}

\author{
H. S. COLLINS
}

Throughout this paper, $S$ will be a compact Hausdorff topological semigroup, $\tilde{S}$ will denote the convolution semigroup of normalized non-negative regular Borel measures on $S$, and $H$ will be the carrier of a measure $\mu$ in $\tilde{S}$. The space of continuous complex valued functions on $S$ will be denoted by $C(S)$, while $C_{R}(S)$ will be the subspace of $C(S)$ of real valued functions. Standard terminology and definitions used here may be found in the references numbered $[2 ; 5]$.

The main result of this paper (Theorem 3 ) is an intrinsic description (in terms of $S, H$, and $C(S)$ ) of the idempotents $\mu$ in $\widetilde{S}$ whose carrier is $H$. Some of the conditions obtained in this theorem are somewhat artificial, and so are not entirely satisfactory. However, the main strength of the theorem is that part which says that the carrier $H$ of each idempotent measure is a compact completely simple semigroup of $S$. This result alone has proved quite useful (e.g., see [1]). Additional useful information contained in Theorem 3 is the statement that the idempotent measure, while not necessarily right invariant on $H$, does have some invariance properties when restricted to each minimal left ideal of $H$.

Preliminary to these theorems we state and prove several lemmas needed in the sequel, the last of which gives a measure theoretic characterization of compact completely simple semigroups. In addition, some additional notation is needed. If $g \in C(S)$ and $x \in S$ let $g^{x}(y)$ $=g(y x)$, so that $g^{x} \in C(S)$. If now $\mu \in \tilde{S}$, define $g^{\prime}(x)=\mu\left(g^{x}\right)$; it is known [2, p. 53] that $g^{\prime} \in C(S)$ as well. It is then clear that a mapping (for fixed $\mu$ ) of $C(S)$ into $C(S)$ is thus defined, and no ambiguity can result in the theorems in which this notation appears if it is understood that these functions $g^{\prime}$ are always defined in terms of a single $\mu$. Occasionally it will be expedient to employ the notation $g^{\prime \prime}$, where by definition $g^{\prime \prime}=\left(g^{\prime}\right)^{\prime}$; i.e., $g^{\prime \prime}(x)=\mu\left[\left(g^{\prime}\right)^{x}\right]$, with $g^{\prime}(t)=\mu\left(g^{t}\right)$.

Lemma 1. Let $A$ and $B$ be subsets of $S$, with $A \subset A^{2}$ and $B$ closed. If $A$ meets the complement of $B$, there exists $c$ in $A$ and non-negative $f$ in $C_{R}(S)$ such that $f(B)=0$ and $f^{c}$ is positive at some point of $A$.

Proof. Let $a \in A \backslash B$. Then $a=b c$, with $b, c \in A$, so by complete regularity there is a non-negative $f$ in $C_{R}(S)$ such that $f(B)=0$ and $1=f(a)=f(b c)=f^{c}(b)$.

Presented to the Society, January 25,1961 ; received by the editors July 18,1960 and, in revised form, May 8, 1961. 
If $H$ is a compact subsemigroup of $S$, the kernel of $H$ is defined as the minimal ideal of $H$. It is known [5] that the minimal left ideals of $H$ are pairwise disjoint and their union is the kernel; a similar statement holds for minimal right ideals. Further, all of these minimal ideals (two-sided, left, or right) are closed sets. Following Wallace, we say $H$ is completely simple if the kernel of $H$ is all of $H$.

Lemma 2. Let $\mu \in \tilde{S}$ and $H=$ carrier $\mu$ be a completely simple semigroup. If $g \in C(S)$ and $g$ is constant on each minimal left ideal of $H$, then $g=g^{\prime}$ on $H$.

Proof. Let $y \in H$. By complete simplicity, there exists a minimal left ideal $L$ of $H$ such that $y \in L=H y$. Then $g^{\prime}(y)=\mu\left(g^{y}\right)=\int_{S} g(x y) d \mu(x)$ $=\int_{H} g(x y) d \mu(x)=\int_{H} g(y) d \mu(x)=g(y) \cdot \mu(H)=g(y)$.

Theorem 1. Let $\mu \in \tilde{S}$ and $H=$ carrier $\mu$. Then $H$ is a completely simple semigroup if and only if (1) $H^{2}=H$ and (2) $f \in C_{R}(S)$ implies $\max f^{\prime}(H)=\max f^{\prime}(K)$, where $K$ is the kernel of $H$.

Proof. Conditions (1) and (2) clearly hold if $H$ is completely simple, for then (since $H^{2}$ is an ideal of $H$ ) $K \subset H^{2} \subset H \subset K$. To complete the proof of the theorem, suppose $H$ is not completely simple. Then $K$ is properly contained in $H=H^{2}$, so by Lemma 1 there exist $a, c \in H$ and non-negative $f$ in $C_{R}(S)$ such that $f(K)=0$, yet $f^{c}(a)>0$. By hypothesis and the remarks preceding Lemma 2 , there is a minimal left ideal $L$ of $H$ and $b \in L$ for which $f^{\prime}(b)=\max f^{\prime}(H) \geqq f^{\prime}(c)>0$. However, $f^{\prime}(b)=\int_{S} f(x b) d \mu(x)=\int_{H} f(x b) d \mu(x)=\int_{H} 0 d \mu(x)=0$, since $b \in L$ implies $H b \subset L \subset K$ and $f(K)=0$. This contradiction completes the proof.

If $H \subset S$, the $H$-translate space is defined as the set of all functions $f^{x}$, where $f \in C(S)$ and $x \in H$, and an element of this set is referred to as an $H$-translate. If $f \in C_{R}(S)$ and $\mu \in \tilde{S}$ with $H=$ carrier $\mu$, the $(\mu, f)$ maximal set is the set of all $x \in H$ for which $\max f^{\prime}(H)=f^{\prime}(x)$.

Theorem 2. Let $\mu \in \tilde{S}$ with $H^{2}=H=$ carrier $\mu$. Then the following are equivalent to one another: (1) $\mu^{2}=\mu$ on the H-translate space, (2) for each $f \in C_{R}(S)$ the $(\mu, f)$ maximal set is a left ideal of $H$, and (3) (i) $H$ is completely simple and (ii) $f \in C(S)$ implies $f^{\prime}$ is constant on each minimal left ideal of $H$.

Proof. We shall prove first that (1) implies (2). If (2) fails, there exist $a, b \in H$ and $f \in C_{R}(S)$ such that $f^{\prime}(b a)<\max f^{\prime}(H)=f^{\prime}(a)$. If $g(x)=f^{\prime}(a)-f^{\prime}(x a)$, then $g \in C_{R}(S), g \geqq 0$ on $H$, and $g(b)>0$, hence $\mu(g)>0$. Thus $0<f^{\prime}(a)-\mu\left[\left(f^{\prime}\right)^{a}\right]=\mu\left(f^{a}\right)-\mu\left[\left(f^{a}\right)^{\prime}\right]=\mu\left(f^{a}\right)-\mu^{2}\left(f^{a}\right)$, which contradicts (1). We next show (2) implies (3). If the kernel $K$ 
of $H$ is a proper subset of $H$, then (since $H^{2}=H$ ) Lemma 1 implies there exist $c \in H$ and non-negative $f \in C_{R}(S)$ such that $f(K)=0$ and $f^{c}>0$ at a point of $H$. Thus $f^{\prime}(c)=\mu\left(f^{c}\right)>0$. Let $a \in H$ be such that $f^{\prime}(a)=\max f^{\prime}(H)$. Then since the $(\mu, f)$ maximal set is a left ideal, $f^{\prime}$ attains its maximum over $H$ at each point of $K a$. Also, if $y \in K a$, $H y \subset H K a \subset K a \subset K$, so that $f^{\prime}(y)=\int_{S} f(x y) d \mu(x)=\int_{H} f(x y) d \mu(x)=0$, since $f(K)=0$. Thus $0=f^{\prime}(y)=\max f^{\prime}(H)=f^{\prime}(a) \geqq f^{\prime}(c)>0$, a contradiction, so $H$ is completely simple. To show each $f^{\prime}$ is constant on each minimal left ideal of $H$, let $L$ be such an ideal. Since $H$ is completely simple, there exists $y \in H$ such that $L=H y$. Assume first that $f \in C_{R}(S)$ and let $b \in H$ be such that $\left(f^{y}\right)^{\prime}(b)=\max \left(f^{y}\right)^{\prime}(H)$; since the $\left(\mu, f^{y}\right)$ maximal set is a left ideal, $H b$ is a subset of it, and $\left(f^{y}\right)^{\prime}=\left(f^{y}\right)^{\prime}(b)$ $=\alpha$ on $H b$. But $H b y \subset H y$ and $H y$ a minimal left ideal together imply $H b y=H y$. Let $z \in H y$. Then $z=h b y$ for some $h \in H$, hence $f^{\prime}(z)$ $=f^{\prime}(h b y)=\left(f^{\prime}\right)^{y}(h b)=\left(f^{y}\right)^{\prime}(h b)=\alpha$; i.e., $f^{\prime}=\alpha$ on $H y$. The extension to $f \in C(S)$ is almost immediate, for if $f=g+i h$, with $g, h \in C_{R}(S)$, and $g=\alpha$ on $L$ and $h=\beta$ on $L$, then $f^{\prime}(y)=\mu\left(f^{y}\right)=\mu\left(g^{y}+i h^{y}\right)=\mu\left(g^{y}\right)+i \mu\left(h^{y}\right)$ $=g^{\prime}(y)+i h^{\prime}(y)=\alpha+i \beta$, for all $y \in L$. To conclude the proof of the theorem, suppose (3) holds. If $g^{t}$ is an $H$-translate, note that $\mu^{2}\left(g^{t}\right)$ $=\mu\left[\left(g^{t}\right)^{\prime}\right]=\mu\left[\left(g^{\prime}\right)^{t}\right]=g^{\prime \prime}(t)$, and since $g^{\prime}$ is constant on each minimal left ideal of $H$, Lemma 2 shows $g^{\prime \prime}(t)=g^{\prime}(t)=\mu\left(g^{t}\right)$. Thus $\mu^{2}=\mu$ on all $H$-translates. This completes the proof.

It should be remarked that the hypothesis $H \subset H^{2}$ was used only in (2) implies (3) of the theorem. However, a very simple example (the closed unit interval of reals with all products equal zero) shows that $\mu^{2}=\mu$ on all $H$-translates does not necessarily imply $H^{2}=H$.

The following four element completely simple semigroup $H=S$ (endowed with the discrete topology) shows that the conditions $H^{2}=H$ and $\mu^{2}=\mu$ on the $H$-translate space do not together imply $\mu^{2}=\mu$ on $C(S)$. Let the multiplication in $S$ be as indicated in the following multiplication table:

\begin{tabular}{c|c|c|c|c} 
& 0 & 1 & 2 & 3 \\
\hline 0 & 0 & 0 & 2 & 2 \\
\hline 1 & 1 & 1 & 3 & 3 \\
\hline 2 & 0 & 0 & 2 & 2 \\
\hline 3 & 1 & 1 & 3 & 3 \\
\hline
\end{tabular}


Here the two minimal left ideals are $\{0,1\}$ and $\{2,3\}$. We regard $C(S)$ as the set of all quadruples $(f(0), f(1), f(2), f(3))$ of complex numbers, and $\widetilde{S}$ as the set of all functions of the form $\phi(x, y, z, w)$ $=a x+b y+c z+d w$, where $a, b, c, d \geqq 0$ and $a+b+c+d=1$. It is easy to compute that if $\mu \in \widetilde{S}$ and $f \in C(S)$ with $\mu=(a, b, c, d)$, then $f^{\prime}(0)$ $=f^{\prime}(1)=a f(0)+b f(1)+c f(0)+d f(1)$ and $f^{\prime}(2)=f^{\prime}(3)=a f(2)+b f(3)$ $+c f(2)+d f(3)$. In particular, $f^{\prime}$ is constant on each minimal left ideal whether $\mu^{2}=\mu$ or not. It is also easy to see that $\mu=(a, b, c, d)$ is idempotent if and only if $(a+b)(a+c)=a,(a+b)(b+d)=b,(c+d)(a+c)$ $=c$, and $(c+d)(b+d)=d$. Finally, if one sets $\mu=(1 / 5,1 / 5,1 / 5,2 / 5)$, then carrier $\mu=H, \mu^{2}=\mu$ on all $H$-translates (here consisting of all the complex numbers $(x, y, x, y))$, yet $\mu^{2} \neq \mu$.

A final remark regarding Theorem 2 may help connect it with previous work. If $S$ is abelian (this was the case Glicksberg considered in [2]), then $\mu^{2}=\mu$ on all $H$-translates and $H^{2}=H$ together imply $\mu^{2}=\mu$. To see this, first note that in this case $H$ is a group since it is all kernel by Theorem 2 and is abelian [3, Corollary 1.2.1]. If $e$ denotes the unit of $H$ and $f \in C(S)$, then $f=f^{e}$ on $H$ and $\mu^{2}(f)=\mu^{2}\left(f^{e}\right)$ $=\mu\left(f^{e}\right)=\mu(f)$, since carrier $\mu=$ carrier $\mu^{2}=H$. A similar argument applies to give the same result in case $H$ is completely simple and has exactly one minimal left ideal (this situation concerned Rosen in [4]), for then $H$ has a right unit $e$.

The next theorem, while far from satisfactory, does give necessary and sufficient conditions that $\mu^{2}=\mu$. It should be noticed that in this theorem we omit the condition $H^{2}=H$ from the hypotheses; in fact, we need not initially even require $H^{2} C H$.

TheOREM 3. If $\mu \in \tilde{S}$ and carrier $\mu=H$, then the following conditions are mutually equivalent: (1) $\mu^{2}=\mu$, (2) $H$ is completely simple; $f \in C(S)$ implies $f^{\prime}$ is constant on each minimal left ideal of $H$; and $\mu^{3}=\mu$, (3) $\mu^{2}=\mu$ on the $H$-translate space and $\mu^{3}=\mu$.

Proof. It is clear by Theorem 2 that (1) implies (2) and (2) implies (3). To prove (3) implies (1), we show first that if (3) holds then $H$ is a semigroup. Note that $\mu^{3}=\mu$ implies [2, Lemma 2.1] $H^{3}=H$, so $H^{2}=H^{4}=\left(H^{2}\right)^{2}$. Thus if $H^{2}$ is not contained in $H$, Lemma 1 applies to give $c \in H^{2}$ and non-negative $f \in C_{R}(S)$ such that $f(H)=0$, yet $f^{c}>0$ at some point of $H^{2}=$ carrier $\mu^{2}$. Thus $\mu^{2}\left(f^{c}\right)>0$. Since $c \in H^{2}$, $a, b \in H$ exist for which $c=a b$, so that if one sets $g(x)=f(x b)$, then $g \in C(S)$ and $g^{a}(x)=g(x a)=f(x a b)=f(x c)=f^{c}(x)$; i.e., $f^{c}$ is an $H$ translate. Now $f^{\prime}(c)=\mu\left(f^{c}\right)=\mu^{2}\left(f^{c}\right)>0$; since in addition $f^{\prime} \geqq 0$ on $H^{2}$ and carrier $\mu^{2}=H^{2}$, we have $\mu^{2}\left(f^{\prime}\right)>0$. However, this says $\mu^{3}(f)>0$, so $\mu(f)>0$. But $f(H)=0$ and carrier $\mu=H$, so $\mu(f)=0$. This contra- 
diction establishes $H^{2} \subset H$, so $H=H^{3}=H^{2} H \subset H H=H^{2}$, and $H^{2}=H$. By Theorem 2, $H$ is completely simple and each $f^{\prime}$ is constant on each minimal left ideal of $H$. But then by Lemma 2 we have that $f^{\prime \prime}=f^{\prime}$ on $H$, all $f \in C(S)$, hence $\mu^{2}(f)=\mu\left(f^{\prime}\right)=\mu\left(f^{\prime \prime}\right)=\mu^{3}(f)=\mu(f)$. This completes the proof.

\section{BIBLIOGRAPHY}

1. H. S. Collins, The kernel of a semigroup of measures, Duke Math. J. 28 (1961), 387-392.

2. I. Glicksberg, Convolution semigroups of measures, Pacific J. Math. 9 (1959), 51-67.

3. R. J. Koch, On topological semigroups, Tulane University Dissertation, 1953.

4. W. G. Rosen, On invariant means over compact semigroups, Proc. Amer. Math. Soc. 7 (1956), 1076-1082.

5. A. D. Wallace, The structure of topological semigroups, Bull. Amer. Math. Soc. 61 (1955), 95-112.

Louisiana State University 